\title{
Nonconforming Galerkin Methods for the Helmholtz Equation
}

\author{
Jim Douglas, Jr.* Juan E. Santos ${ }^{\dagger} \quad$ Dongwoo Sheen ${ }^{\ddagger}$
}

January 29, 2001

\begin{abstract}
Nonconforming Galerkin methods for a Helmholtz-like problem arising in seismology are discussed both for standard simplicial linear elements and for several new rectangular elements related to bilinear or trilinear elements. Optimal order error estimates in a broken energy norm are derived for all elements and in $L^{2}$ for some of the elements when proper quadrature rules are applied to the absorbing boundary condition. Domain decomposition iterative procedures are introduced for the nonconforming methods, and their convergence at a predictable rate is established.
\end{abstract}

Subject Classification: Primary 65N30; Secondary

Keywords: nonconforming finite element, Helmholtz, domain decomposition method

\section{Introduction}

Seismic waves attenuate when travelling through rocks and other solid materials, with the fraction of energy loss per cycle being essentially independent of frequency over a wide range of frequencies and with attenuation being an increasing function of frequency. These attenuation effects are more often described better in the spacefrequency domain than in the space-time domain, which leads to the formulation of a Helmholtz-like problem to describe the behavior of seismic waves at a given angular frequency. For the computational purpose, the medium is usually truncated into a bounded domain of reasonable size for computation and with artificial boundaries on

${ }^{*}$ Center for Applied Mathematics, Purdue University, West Lafayette, IN 47907-1395, U.S.A. Email: douglas@math.purdue.edu.

${ }^{\dagger}$ Center for Applied Mathematics, Purdue University, West Lafayette, IN 47907-1395, U.S.A., and CONICET, Observatorio Astronómico, Universidad Nacional de La Plata, La Plata (1900), Argentina. E-mail: santos@math.purdue.edu.

${ }^{\ddagger}$ Department of Mathematics, Seoul National University, Seoul 151-747, Korea; supported in part by GARC and KOSEF 97-0701-01-01-3, 961-0106-039-2. E-mail: sheen@math.snu.ac.kr. 
which absorbing boundary conditions are employed to minimize the effects of these boundaries.

The Helmholtz-like problem to be considered in the paper is to describe pressure waves in a two- or three-dimensional bounded domain with an absorbing boundary condition. The object of this paper is to present a collection of nonconforming Galerkin procedures and corresponding domain decomposition iterative procedures to solve the problem. Analyses will be carried out for nonconforming methods based on the triangulation of the domain into $N$-simplices [7] or $N$-rectangles [12] for $N=2$ or 3 . An extension to quadrilaterals can be made for $N=2$.

Nonconforming finite element methods have been employed by structural engineers since about 1960: for instance, see the elements of Adini [1], Morley [19], Zienkiewicz [25]. Also for elliptic cases including Stokes and elasticity problems, well-known are the $P_{1}$ nonconforming element of Crouzeix and Raviart [7] and the rotated $Q_{1}$ element of Rannacher and Turek [20]. In our recent papers [12, 5], a modification to the rotated $Q_{1}$ element has been made to have simple degrees of freedom for second-order elliptic problems.

Among the advantages of using our nonconforming elements instead of standard conforming ones, we mention that the hybridization of nonconforming procedures is easier to achieve. Specifically, the Lagrange multipliers associated with fluxes on each inter-element boundary are constants with the degrees of freedom being the values at the midpoints of boundaries, whereas in the case of conforming elements the number of dof's is equal to the number of vertices of the face of the element. Consequently, the amount of information to be passed to the neighboring subdomains is considerably reduced if nonconforming elements are used in domain decomposition procedures. Another advantage of using our nonconforming elements is that the spectral radius of the iteration operator in the domain decomposition iterative procedure can be estimated, and that has not been done for conforming elements.

In $\S 3, L^{2}(\Omega)$ and $H^{1}(\Omega)$ error estimates for the global nonconforming Galerkin method are derived. Since the bilinear form related with Helmholtz-like problem is noncoercive, it does not determines a norm and, consequently, the Strang lemmas [23, 24] do not hold. Therefore, a bootstrapping argument of Schatz [22] used by DouglasSantos-Sheen-Bennethum in [10] to analyze a similar problem using conforming finite element spaces will be applied.

The global hybridization of the nonconforming procedure and a corresponding domain decomposition iterative procedure will be described and analyzed in $\S 4$ and $\S 5$, respectively. Quite analogous iterative procedures for nonconforming methods for coercive second order elliptic problems were introduced by Douglas et. al., [12] and were based on ideas for conforming methods for second order elliptic problems introduced first by P. L. Lions $[16,17]$ and then applied to the more difficult Helmholtz problem by Després [8]; later [9], a more precise convergence argument was given for the coercive second order elliptic problem as approximated by mixed finite element methods.

The organization of the paper is as follows. In $\S 2$ our model problem is stated. In $\S 3$ a global nonconforming Galerkin method is defined and optimal order error estimates 
are derived. This global nonconforming Galerkin method is hybridized in the next section. The convergence and spectral radius of the domain decomposition iteration are studied in detail in $\S 5$. In the final section we prove some technical lemmata.

\section{The Helmholtz Problem}

\subsection{The Model Problem}

Let $\Omega=(0,1)^{N}, N=2$ or 3 , and $\Gamma=\partial \Omega$, and let $\nu$ denote the unit outward normal to $\Gamma$. Given $f(\cdot, \omega) \in H^{-1}(\Omega)$ for a fixed $\omega>0$, consider the following Helmholtz problem:

$$
\begin{aligned}
L u=-\frac{\omega^{2}}{K(x, \omega)} u-\nabla \cdot\left(\frac{1}{\rho(x)} \nabla u\right)=f(x, \omega), & x \in \Omega, \\
\frac{\partial u}{\partial \nu}+i \omega \alpha(x, \omega) u=0, & x \in \Gamma,
\end{aligned}
$$

where $K(\cdot, \omega), \rho \in L^{\infty}(\Omega)$, and $\alpha(\cdot, \omega) \in L^{\infty}(\Gamma)$, along with some additional assumptions to be specified later. In (2.1), $u(x, \omega)$ represents the Fourier transform (in time) of the pressure $u(x, t)$ and $\rho(x)$ is the density, assumed to be bounded below and above by positive quantities $\rho_{\min }$ and $\rho_{\max }$, respectively. Also,

$$
K(x, \omega)=K_{r}(x, \omega)+i K_{i}(x, \omega)=\frac{K R(x)}{\beta(x, \omega)-i \gamma(x, \omega)}
$$

is the complex bulk modulus of the viscoacoustic material. The real and imaginary parts of $K(x, \omega)$ are related by the quality factor $Q(x, \omega)$ :

$$
\frac{1}{Q(x, \omega)} \approx \frac{\gamma(x, \omega)}{\beta(x, \omega)} .
$$

The coefficients $\beta(x, \omega)$ and $\gamma(x, \omega)$ characterize the dispersive properties of the material and will be chosen to be of the form (see [18, 21])

$$
\begin{aligned}
& \beta(x, \omega)=1-\frac{1}{\pi Q_{m}(x)} \ln \frac{1+\omega^{2} \tau_{1}^{2}(x)}{1+\omega^{2} \tau_{2}^{2}(x)}, \\
& \gamma(x, \omega)=\frac{2}{\pi Q_{m}(x)} \tan ^{-1} \frac{\omega\left(\tau_{1}(x)-\tau_{2}(x)\right)}{1+\omega^{2} \tau_{1}(x) \tau_{2}(x)}
\end{aligned}
$$

$\tau_{1}(x)$ and $\tau_{2}(x)$ are given angular frequencies such that the quality factor $Q(x, \omega)$ is approximately equal to a constant $Q_{m}(x)$ in the range $\tau_{1}^{-1}(x) \leq \omega \leq \tau_{2}^{-1}(x)$. Realistic values for $Q_{m}(x)$ in rocks are in the range 30 to 1000 .

Equation (2.1.b) is a first-order absorbing boundary condition obtained by imposing the condition that the boundary $\Gamma$ be transparent for normally arriving waves. Its derivation can be found in [21]. The complex coefficient $\alpha(x, \omega)$ can be written as

$$
\alpha(x, \omega)=M(x, \omega)-i N(x, \omega),
$$


with $M(x, \omega)$ and $N(x, \omega)$ being given by

$$
\begin{aligned}
& \text { i) } M(x, \omega)=C_{r}\left(2\left(C_{r}^{4}+C_{i}^{4}\right)\right)^{-1 / 2}\left[1+\left(1+\left(C_{i} / C_{r}\right)^{4}\right)^{1 / 2}\right]^{1 / 2}, \\
& \text { ii) } \quad N(x, \omega)=\frac{C_{i}^{2}}{C_{r}}\left(2\left(C_{r}^{4}+C_{i}^{4}\right)\right)^{-1 / 2}\left[1+\left(1+\left(C_{i} / C_{r}\right)^{4}\right)^{1 / 2}\right]^{-1 / 2},
\end{aligned}
$$

and

$$
C_{r}^{2}(x, \omega)=K_{r}(x, \omega) / \rho(x), \quad C_{i}^{2}(x, \omega)=K_{i}(x, \omega) / \rho(x) .
$$

Set

$$
-\frac{\omega^{2}}{K(x, \omega)}=-A(x, \omega)+i B(x, \omega), \quad i \omega \frac{\alpha(x, \omega)}{\rho(x)}=C(x, \omega)+i D(x, \omega),
$$

and assume that $A(x, \omega), B(x, \omega), C(x, \omega)$, and $D(x, \omega)$ are bounded below and above by positive constants. Let $B_{\min }=B_{\min }(\omega)$ be the lower bound for $B(x, \omega)$.

\subsection{Weak formulation}

Set

$$
a(u, v)=-\omega^{2}\left(\frac{1}{K} u, v\right)+\left(\frac{1}{\rho} \nabla u, \nabla v\right)+i \omega\left\langle\frac{\alpha}{\rho} u, v\right\rangle_{\Gamma}
$$

where $(\cdot, \cdot)$ and $\langle\cdot, \cdot\rangle_{\Gamma}$ denote complex $L^{2}(\Omega)$ and $L^{2}(\Gamma)$ inner products, respectively. A weak formulation of (2.1) is to find $u \in H^{1}(\Omega)$ such that

$$
a(u, v)=(f, v), \quad v \in H^{1}(\Omega) .
$$

Minor modifications of the argument given in $[11,15]$ lead to the following theorem.

Theorem 2.1 For $\omega \neq 0$ and $f(\cdot, \omega) \in H^{-1}(\Omega)$, there exists a unique solution $u(\cdot, \omega) \in$ $H^{1}(\Omega)$ to (2.6), and it is also a unique solution to (2.1). Moreover, if $f(\cdot, \omega) \in L^{2}(\Omega)$, then $u(\cdot, \omega) \in H^{2}(\Omega)$.

\section{The Nonconforming Galerkin Method}

We shall approximate the solution of (2.6) using nonconforming finite elements based on standard simplicial elements or the rectangular elements given in [12]. For $h>0$, let $\mathcal{T}_{h}$ be a quasiregular triangulation of $\bar{\Omega}$ such that $\bar{\Omega}=\cup_{j=1}^{J} \bar{\Omega}_{j}$ with $\Omega_{j}$ being $N$ simplices or $N$-rectangles of $\operatorname{diam}\left(\Omega_{j}\right) \leq h$. Set

$$
\Gamma_{j}=\partial \Omega \cap \partial \Omega_{j}, \quad \Gamma_{j k}=\Gamma_{k j}=\partial \Omega_{j} \cap \partial \Omega_{k},
$$

and denote by $\xi_{j}$ and $\xi_{j k}$ the centroids of $\Gamma_{j}$ and $\Gamma_{j k}$, respectively. 


\subsection{Nonconforming elements}

For the simplicial nonconforming elements, set (as usual)

$$
\mathcal{N C}_{j}^{h}=\mathcal{P}_{1}\left(\Omega_{j}\right),
$$

where $\mathcal{P}_{\ell}(E)$ is the class of polynomials of degree $\ell$ on the set $E$. For the rectangular nonconforming elements, we shall make use of the same elements as described in [12]. Let

$$
\theta_{\ell}(x)= \begin{cases}x^{2}-\frac{5}{3} x^{4}, & \ell=1, \\ x^{2}-\frac{25}{6} x^{4}+\frac{7}{2} x^{6}, & \ell=2,\end{cases}
$$

and, in the two-dimensional case, define two reference bases by

$$
\mathcal{Q}_{\ell}=\operatorname{Span}\left\{1, x, y, \theta_{\ell}(x)-\theta_{\ell}(y)\right\}, \quad \ell=1,2,
$$

on the reference element $\widehat{R}=[-1,1]^{2}$. A nodal basis is easily found; the basis function corresponding to the node $(1,0)$ is given by

$$
w_{1,0}^{(\ell)}(x, y)=\frac{1}{4}+\frac{1}{2} x+\frac{\theta_{\ell}(x)-\theta_{\ell}(y)}{4 \theta_{\ell}(1)}, \quad \ell=1,2 .
$$

The method can be adapted to allow quadrilaterals that are not parallelograms in the partition. In this case the basis on the reference square should be modified to include the term $x y$ just for these quadrilaterals; see $[2,5]$

For the three-dimensional case with $\widehat{R}=[-1,1]^{3}$, the minimum dimension of $\mathcal{Q}_{\ell}$ is six, and the choices

$$
\begin{aligned}
\mathcal{Q}_{\ell} & =\operatorname{Span}\left\{1, x, y, z, \theta_{\ell}(x)-\theta_{\ell}(y), \theta_{\ell}(x)-\theta_{\ell}(z)\right\} \\
& =\operatorname{Span}\left\{1, x, y, z, \theta_{\ell}(y)-\theta_{\ell}(z), \theta_{\ell}(y)-\theta_{\ell}(x)\right\} \\
& =\operatorname{Span}\left\{1, x, y, z, \theta_{\ell}(z)-\theta_{\ell}(x), \theta_{\ell}(z)-\theta_{\ell}(y)\right\}, \quad \ell=1,2,
\end{aligned}
$$

have that dimension. The nodal basis element associated with the node $(1,0,0)$ is given by

$$
w_{1,0,0}^{(\ell)}(x, y, z)=\frac{1}{6}+\frac{1}{2} x-\frac{1}{6 \theta_{\ell}(1)}\left(2 \theta_{\ell}(x)-\theta_{\ell}(y)-\theta_{\ell}(z)\right), \quad \ell=1,2 ;
$$

the other five nodal basis functions can be obtained by reflection and permutation. Two other acceptable choices are given by

$$
\begin{aligned}
\mathcal{Q}_{\ell} & =\operatorname{Span}\left\{1 ; x_{i}, \theta_{\ell}\left(x_{i}\right), i=1,2,3\right\} \\
& =\operatorname{Span}\left\{\frac{1}{2} x_{i} \pm \frac{\theta_{\ell}\left(x_{i}\right)}{2 \theta_{\ell}(1)}, i=1,2,3 ; 1-\frac{1}{\theta_{\ell}(1)} \sum_{i=1}^{3} \theta_{\ell}\left(x_{i}\right)\right\} \quad \ell=1,2
\end{aligned}
$$


The seven degrees of freedom associated with (3.5) are the values at the centers of the faces and at the center of the element; for computational purposes, the basis element associated to the origin is a bubble function (as shown above) and can be eliminated without serious cost over what would be required with the corresponding basis consisting of six functions.

For the rectangular nonconforming elements in two or three dimension, let

$$
\mathcal{N C}_{j}^{h}=\mathcal{Q}_{\ell}\left(\Omega_{j}\right), \quad \ell=1,2 .
$$

The simplicial or rectangular nonconforming finite element space is then defined as follows:

$$
\mathcal{N C}^{h}=\left\{v\left|v_{j}:=v\right|_{\Omega_{j}} \in \mathcal{N C}_{j}^{h}, j=1, \ldots, J ; v_{j}\left(\xi_{j k}\right)=v_{k}\left(\xi_{j k}\right), \forall\{j, k\}\right\},
$$

with the degrees of freedom taken to be values at the midpoints $\xi_{j k}$ of each faces of $\Omega_{j}$, and in the case (3.5) with additional value at the center of the element.

Note that all of the simplicial and rectangular elements given above have the critical orthogonality property [12]

$$
\left\langle 1, w_{j}-w_{k}\right\rangle_{\Gamma_{j k}}=0, \quad w \in \mathcal{N C}^{h}
$$

Moreover, in the rectangular case, $\mathcal{Q}_{\ell}(\widehat{R})$ is invariant under both reflection and permutation of the coordinates. For $\ell=2$, if $v_{j} \in \mathcal{N C}_{j}^{h}$ vanishes at a midpoint $\xi_{j}$ of the boundary face $\Gamma_{j}$, so does the approximate integral by quadrature at the two-point or $2 \times 2$-point Gauss rule on $\Gamma_{j}$; this property is valuable in establishing optimal order convergence of the numerical solution in $L^{2}(\Omega)$. Thus, all of these choices (for $\ell=1$ or 2) for a local basis are acceptable and are essentially indistinguishable with respect to difficulty of assembly of the approximate problem and the solution of the associated linear equations, though the numerical solutions will differ.

When $N=2$, quadrilateral elements can be employed without difficulty; see [12] for details which are equally applicable here.

\subsection{The nonconforming Galerkin method}

Let $(\cdot, \cdot)_{j}=(\cdot, \cdot)_{\Omega_{j}}$, and set, for $u, v \in L^{2}(\Omega)$ such that $u_{j}, v_{j} \in H^{1}\left(\Omega_{j}\right)$,

$$
\begin{aligned}
a_{h}(u, v) & =-\left(\frac{\omega^{2}}{K} u, v\right)+\sum_{j}\left(\frac{1}{\rho} \nabla u, \nabla v\right)_{j}+i \omega\left\langle\left\langle\frac{\alpha}{\rho} u, v\right\rangle\right\rangle_{\Gamma} \\
& =\sum_{j}(L u, v)_{j}+\sum_{j}\left\langle\frac{1}{\rho} \frac{\partial u}{\partial \nu}, v\right\rangle_{\partial \Omega_{j}}+i \omega\left\langle\left\langle\frac{\alpha}{\rho} u, v\right\rangle\right\rangle_{\Gamma} .
\end{aligned}
$$

The nonconforming Galerkin approximation of (2.1) is defined as $u^{h} \in \mathcal{N C}^{h}$ such that

$$
a_{h}\left(u^{h}, v\right)=(f, v), \quad v \in \mathcal{N C}^{h}
$$


In $(3.7),\langle\langle\cdot, \cdot\rangle\rangle$ denotes an approximation of $\langle\cdot, \cdot\rangle$ on the boundary faces by a quadrature rule. In order to define specific quadrature rules to follow, we shall assume that $f \in L^{2}(\Omega)$ so that the solution $u$ of $(2.6)$ to belong to $H^{2}(\Omega)$. However, if the boundary integrals are evaluated exactly, such an assumption will not be necessary, at least in defining the nonconforming procedure (3.8). The first-order correct mid-point rule will be employed for all three types of elements: simplices, 2-quadrilaterals, and 3rectangles. We shall also employ second-order correct rules, which will be different depending on the type of element to be treated. For the two-dimensional cases, the boundary faces are lines and the usual two-point Gauss quadrature rules will suffice. For 3-simplicial elements, if $T$ is a boundary triangle, let $\delta_{i}, i=1,2,3$, be the midpoints of the edges of $T$ and set

$$
\langle\langle v, w\rangle\rangle_{T}=\sum_{i=1}^{3}(v \bar{w})\left(\delta_{i}\right) \frac{|T|}{3} .
$$

This quadrature rule ([6, p.183]) is exact on polynomials of degree two. For 3-rectangular elements, we shall use $2 \times 2$ Gauss quadrature on each face of a 3-rectangular element contained in $\Gamma$.

To show uniqueness of the solution $u^{h}$ of (3.8), set $f=0$ in (3.8) and choose $v=u^{h}$. Taking the imaginary part in the resulting equation and using the fact that $M>0$ and $K_{i}>0$, we immediately conclude that $u^{h}$ vanishes. Existence follows from finite dimensionality.

\subsection{Error estimates}

Next, we shall derive error estimates for the procedure (3.8). Let broken norms and seminorms be defined by

$$
\|u\|_{m, h}^{2}=\sum_{j}\|u\|_{m, \Omega_{j}}^{2}, \quad|u|_{m, h}^{2}=\sum_{j}|u|_{m, \Omega_{j}}^{2}, \quad|u|_{m, h, \Gamma}^{2}=\sum_{j}|u|_{m, \Gamma_{j}}^{2} .
$$

Also, set

$$
\widetilde{\Lambda}^{h}=\left\{\lambda \in \Pi_{j, k} \mathcal{P}_{0}\left(\Gamma_{j k}\right): \lambda_{j k} \in \mathcal{P}_{0}\left(\Gamma_{j k}\right) ; \lambda_{j k}+\lambda_{k j}=0 \forall j, k\right\}
$$

Let us define projections $\Pi$ and $P_{0}$ by

$$
\begin{array}{llc}
\Pi: H^{2}(\Omega) \rightarrow \mathcal{N C}^{h}: & (v-\Pi v)(\xi)=0, & \xi=\xi_{j k} \text { or } \xi_{j} ; \\
P_{0}: H^{2}(\Omega) \rightarrow \widetilde{\Lambda}^{h}: & \left\langle\frac{1}{\rho} \frac{\partial v_{j}}{\partial \nu_{j}}-P_{0} v_{j}, z\right\rangle_{\gamma}=0, & z \in \mathcal{P}_{0}(\gamma), \quad \gamma=\Gamma_{j k} \text { or } \Gamma_{j} ;
\end{array}
$$

if a seven dimensional basis is employed on $\Omega_{j}$, add equality at the center of $\Omega_{j}$ to the requirements for $\Pi$. 
Since $\Pi$ reproduces linear functions on elements and $P_{0}$ reproduces constants on faces, it follows from standard polynomial approximation results $[4,6]$ that

$$
\begin{aligned}
& \|v-\Pi v\|^{2}+h^{2} \sum_{j}\|v-\Pi v\|_{1, j}^{2}+h^{4} \sum_{j}\|v-\Pi v\|_{2, j}^{2}+h \sum_{j}|v-\Pi v|_{0, \partial \Omega_{j}}^{2} \\
& \quad+h^{3} \sum_{j}\left|\frac{\partial}{\partial \nu_{j}}(v-\Pi v)\right|_{0, \partial \Omega_{j}}^{2}+h^{3} \sum_{j}\left|\frac{1}{\rho} \frac{\partial v_{j}}{\partial \nu_{j}}-P_{0} v\right|_{0, \partial \Omega_{j}}^{2} \\
& \leq C\|v\|_{2}^{2} h^{4}, \quad v \in H^{2}(\Omega) .
\end{aligned}
$$

Denote by $E(G, w)$ the boundary quadrature error:

$$
E(v, w)=\sum_{j}\left\{\langle v, w\rangle_{\Gamma_{j}}-\langle\langle v, w\rangle\rangle_{\Gamma_{j}}\right\}
$$

The following bounds for the boundary quadrature errors will be used in the error analysis that follows. If the subscript $\ell$ is used to indicate the order of the rule for simplicial elements and both the index for $\mathcal{Q}_{\ell}$ and the order of the quadrature rule for rectangular elements, it is shown in [12] that

$$
\begin{array}{ll}
\left|E_{\ell}(\varphi, w)\right| \leq C h|\varphi|_{1, \partial \Omega}|w|_{\partial \Omega}, & w \in \mathcal{N C}^{h}, \quad \ell=1 \text { or } 2, \\
\left|E_{2}(\varphi, w)\right| \leq C h^{2}|\varphi|_{2, \partial \Omega}|w|_{\partial \Omega}, & w \in \mathcal{N C}^{h} .
\end{array}
$$

Also, it is shown in the appendix $(\S 6)$ that

$$
\left|E_{\ell}(\varphi, w)\right| \leq C h\|\varphi\|_{1, h}\|w\|_{1, h}, \quad \varphi, w \in \mathcal{N C}^{h}, \quad \ell=1 \text { or } 2 .
$$

Set

$$
\delta=u-u^{h}, \quad \eta=\Pi u-u^{h} .
$$

Then, by (3.7) and the orthogonality of $v \in \mathcal{N C}^{h}$ on any edge to constants,

$$
a_{h}(\delta, v)=\sum_{j}\left\langle\frac{1}{\rho} \frac{\partial u_{j}}{\partial \nu_{j}}-P_{0} u_{j}, v\right\rangle_{\partial \Omega_{j} \backslash \Gamma_{j}}-i \omega E\left(\frac{\alpha}{\rho} u, v\right), \quad v \in \mathcal{N C}^{h} .
$$

Note that since neither $a(\cdot, \cdot)$ nor $a_{h}(\cdot, \cdot)$ is coercive, neither $a(u, u)$ nor $a_{h}(u, u)$ determines a norm. Consequently, the Strang lemmas do not hold (see $[6,23,24]$ ). Therefore, we follow the argument of Schatz used by Douglas-Santos-Sheen-Bennethum in [10] to analyze a similar problem using conforming finite element spaces. First, we will obtain an estimate of $\|\eta\|_{0}$ in terms of $\|\eta\|_{1, h}$ and $\|u\|_{2}$, which in turn will imply an estimate of $\|\delta\|_{0}$ in terms of $\|\delta\|_{1, h}$ and $\|u\|_{2}$. Then, a bootstrapping argument will be applied to obtain $L^{2}(\Omega)$ and $H^{1}(\Omega)$ error estimates in terms of $\|u\|_{2}$.

Start by considering the dual problem to (2.1) to find $\psi \in H^{2}(\Omega)$ satisfying

$$
\begin{aligned}
L^{*} \psi:=-\frac{\omega^{2}}{\bar{K}} \psi-\nabla \cdot\left(\frac{1}{\rho} \nabla \psi\right) & =\eta, & & x \in \Omega, \\
\frac{\partial \psi}{\partial \nu}-i \omega \bar{\alpha} \psi & =0, & & x \in \Gamma,
\end{aligned}
$$


By standard elliptic regularity results for second order elliptic equations,

$$
\|\psi\|_{2} \leq C(\omega)\|\eta\|_{0} .
$$

First, by (3.6), for $q_{j} \in P_{0}\left(\Omega_{j}\right)$ we have

$$
\begin{aligned}
\|\eta\|_{0}^{2} & =\left(\eta, L^{*} \psi\right) \\
& =-\omega^{2}\left(\frac{1}{K} \eta, \psi\right)+\sum_{j}\left(\frac{1}{\rho} \nabla \eta, \nabla \psi_{j}\right)-\sum_{j}\left\langle\eta, \frac{1}{\rho} \frac{\partial \psi}{\partial \nu}\right\rangle_{\partial \Omega_{j} \backslash \Gamma}+i \omega\left\langle\frac{\alpha}{\rho} \eta, \psi\right\rangle_{\Gamma} \\
& =a_{h}(\eta, \psi)+i \omega E\left(\frac{\alpha}{\rho} \eta, \psi\right)-\sum_{j}\left\langle\eta, \frac{1}{\rho} \frac{\partial \psi}{\partial \nu}\right\rangle_{\partial \Omega_{j} \backslash \Gamma} \\
& =a_{h}(\eta, \psi)+i \omega E\left(\frac{\alpha}{\rho} \eta, \psi\right)-\sum_{j}\left\langle\eta_{j}-q_{j}, \frac{1}{\rho} \frac{\partial \psi_{j}}{\partial \nu_{j}}-P_{0} \psi_{j}\right\rangle_{\partial \Omega_{j} \backslash \Gamma} .
\end{aligned}
$$

Next, for $v \in \mathcal{N} \mathcal{C}^{h}$,

$$
\begin{aligned}
a_{h}(\eta, v) & =a_{h}(\delta, v)-a_{h}(u-\Pi u, v) \\
& =\sum_{j}\left\langle\frac{1}{\rho} \frac{\partial u_{j}}{\partial \nu_{j}}-P_{0} u_{j}, v\right\rangle_{\partial \Omega_{j} \backslash \Gamma}-i \omega E\left(\frac{\alpha}{\rho} u, v\right)-a_{h}(u-\Pi u, v) .
\end{aligned}
$$

From (3.15) and (3.16),

$$
\begin{aligned}
\|\eta\|_{0}^{2}=a_{h}(\eta, \psi-v)-a_{h}(u-\Pi u, v)+i \omega\left[E\left(\frac{\alpha}{\rho} \eta, \psi\right)-E\left(\frac{\alpha}{\rho} u, v\right)\right] & \\
- & \sum_{j}\left\langle\eta_{j}-q_{j}, \frac{1}{\rho} \frac{\partial \psi_{j}}{\partial \nu_{j}}-P_{0} \psi_{j}\right\rangle_{\partial \Omega_{j} \backslash \Gamma}+\sum_{j}\left\langle\frac{1}{\rho} \frac{\partial u_{j}}{\partial \nu_{j}}-P_{0} u_{j}, v_{j}-\psi_{j}\right\rangle_{\partial \Omega_{j} \backslash \Gamma} .
\end{aligned}
$$

Let us bound each term on the right side of (3.17). Thanks to (3.9) and (3.15), $v \in \mathcal{N C}^{h}$ can be chosen such that

$$
\|\psi-v\|_{0}+h\|\psi-v\|_{1, h}+h^{2}\|v\|_{2, h}+h^{1 / 2}|\psi-v|_{0, \Gamma} \leq C\|\psi\|_{2} h^{2} \leq C\|\eta\|_{0} h^{2} .
$$

Then,

$$
\begin{aligned}
\left|a_{h}(\eta, \psi-v)\right| & \leq C(\omega)\left[\|\eta\|_{1, h}\|\psi-v\|_{1, h}+\left|\langle\langle\eta, \psi-v\rangle\rangle_{\Gamma}\right|\right] \\
& \leq C(\omega)\left[h\|\eta\|_{1, h}\|\eta\|_{0}+\left|\langle\eta, \psi-v\rangle_{\Gamma}\right|+\omega|E(\eta, \psi-v)|\right] \\
& \leq C(\omega)\left[h\|\eta\|_{1, h}\|\eta\|_{0}+|\eta|_{0, \Gamma}|\psi-v|_{0, \Gamma}+|\eta|_{0, \Gamma}|\psi-v|_{1, h, \Gamma} h\right] \\
& \leq C(\omega) h\|\eta\|_{1, h}\|\eta\|_{0},
\end{aligned}
$$

by (3.18), elliptic regularity (3.14), the trace inequality, and (3.10). 
The second term can be decomposed as follows:

$$
\begin{aligned}
& a_{h}(u-\Pi u, v) \\
& =\sum_{j}\left(u-\Pi u, L^{*} v\right)_{j}+\sum_{j}\left\langle u-\Pi u, \frac{1}{\rho} \frac{\partial v_{j}}{\partial \nu_{j}}\right\rangle_{\partial \Omega_{j}}+i \omega\left\langle\left\langle\frac{\alpha}{\rho}(u-\Pi u), v\right\rangle\right\rangle_{\Gamma} \\
& =\sum_{j}\left(u-\Pi u, L^{*} v\right)_{j}+\sum_{j}\left\langle u-\Pi u, \frac{1}{\rho} \frac{\partial v_{j}}{\partial \nu_{j}}\right\rangle_{\partial \Omega_{j} \backslash \Gamma} \\
& \quad-\sum_{j}\left\langle u-\Pi u,\left(\frac{1}{\rho} \frac{\partial}{\partial \nu}-i \omega \frac{\bar{\alpha}}{\rho}\right)\left(\psi_{j}-v_{j}\right)\right\rangle_{\Gamma_{j}}-i \omega E\left(\frac{\alpha}{\rho}(u-\Pi u), v\right),
\end{aligned}
$$

where the boundary condition (3.13b) was invoked. Let us obtain an estimate for each term in the right side of (3.20). First note that the argument given in [12] implies that

$$
\left|\sum_{j}\left(u-\Pi u, L^{*} v\right)_{j}\right|+\left|\sum_{j}\left\langle u-\Pi u, \frac{1}{\rho} \frac{\partial v_{j}}{\partial \nu_{j}}\right\rangle_{\partial \Omega_{j} \backslash \Gamma}\right| \leq C\|u\|_{2}\|\eta\|_{0} h^{2} .
$$

The third term in (3.20) can be estimated as follows:

$$
\begin{aligned}
& \left|\sum_{j}\left\langle u-\Pi u,\left(\frac{1}{\rho} \frac{\partial}{\partial \nu}-i \omega \frac{\bar{\alpha}}{\rho}\right)\left(\psi_{j}-v_{j}\right)\right\rangle_{\Gamma_{j}}\right| \\
& \quad \leq C(\omega)|u-\Pi u|_{0, \Gamma}\|\psi-v\|_{1, h}^{1 / 2}\|\psi-v\|_{2, h}^{1 / 2} \leq C(\omega)\|u\|_{2}\|\eta\|_{0} h^{2} .
\end{aligned}
$$

By (3.10), the last term in (3.20) can be bounded by

$$
\omega\left|E_{\ell}\left(\frac{\alpha}{\rho}(u-\Pi u), v\right)\right| \leq \begin{cases}C(\omega)\|u\|_{2}\|\eta\|_{0} h^{3 / 2}, & \ell=1 \text { or } 2 \\ C(\omega)|u|_{2, \Gamma}\|\eta\|_{0} h^{2}, & \ell=2 .\end{cases}
$$

Thus, (3.21)-(3.23) imply that the second term in (3.17) can be bounded as follows:

$$
\left|a_{h}(u-\Pi u, v)\right| \leq C(\omega)\|\eta\|_{0}\left[\|u\|_{2} h^{2}+\varepsilon_{\ell}\right],
$$

where

$$
\varepsilon_{\ell} \leq \begin{cases}\|u\|_{2} h, & \ell=1 \text { or } 2 \\ |u|_{2, \Gamma} h^{2}, & \ell=2\end{cases}
$$

Now, we continue to bound the other terms in the right side of (3.17). For the third term, using (3.10), we have

$$
\left|i \omega E_{\ell}\left(\frac{\alpha}{\rho} \eta, \psi\right)\right| \leq C(\omega)|\eta|_{0, \Gamma}|\psi|_{1, \Gamma} h \leq C(\omega)\|\eta\|_{1, h}\|\eta\|_{0} h, \quad \ell=1 \text { or } 2,
$$


and

$$
\left|i \omega E_{\ell}\left(\frac{\alpha}{\rho} u, v\right)\right| \leq \begin{cases}C(\omega)|u|_{1, \Gamma}|v|_{0, \Gamma} h \leq C(\omega)\|u\|_{2}\|\eta\|_{0} h, & \ell=1 \text { or } 2 \\ C(\omega)|u|_{2, \Gamma}|v|_{0, \Gamma} h^{2} \leq C(\omega)|u|_{2, \Gamma}\|\eta\|_{0} h^{2}, & \ell=2 .\end{cases}
$$

Next, for properly chosen $q$, the fourth term has the bound (see [12])

$$
\left|\sum_{j}\left\langle\eta_{j}-q_{j}, \frac{1}{\rho} \frac{\partial \psi_{j}}{\partial \nu_{j}}-P_{0} \psi_{j}\right\rangle_{\partial \Omega_{j} \backslash \Gamma}\right| \leq C\|\eta\|_{0}\|\eta\|_{1, h} h .
$$

Finally, for the last term in the right side of (3.17) we have

$$
\left|\sum_{j}\left\langle\frac{1}{\rho} \frac{\partial u_{j}}{\partial \nu_{j}}-P_{0} u_{j}, v_{j}-\psi_{j}\right\rangle_{\partial \Omega_{j} \backslash \Gamma}\right| \leq C\|u\|_{2}\|\eta\|_{0} h^{2} .
$$

By applying (3.20) and (3.24)-(3.29) in (3.17), we conclude that

$$
\|\eta\|_{0} \leq C(\omega)\left[\|\eta\|_{1, h} h+\|u\|_{2} h^{2}+\varepsilon_{\ell}\right] .
$$

Next, we bound $\|\delta\|_{0}$ using the triangle inequality, (3.30), and an approximation property of $\Pi$ :

$$
\begin{aligned}
\|\delta\|_{0} & \leq\|\eta\|_{0}+\|u-\Pi u\|_{0} \leq C(\omega)\left[h\|\eta\|_{1, h}+h^{2}\|u\|_{2}+\varepsilon_{\ell}\right] \\
& \leq C(\omega)\left[h\left(\|\delta\|_{1, h}+\|u-\Pi u\|_{1, h}\right)+h^{2}\|u\|_{2}+\varepsilon_{\ell}\right] \leq C(\omega)\left[h\|\delta\|_{1, h}+h^{2}\|u\|_{2}+\varepsilon_{\ell}\right] .
\end{aligned}
$$

Next, we wish to bound $\|\delta\|_{1, h}$ in terms of $\|\delta\|_{0}$ and $\|u\|_{2}$. First, by (3.7) and (3.12),

$$
\begin{aligned}
& \frac{1}{\rho_{\min }}\|\delta\|_{1, h}^{2} \leq\left\|\frac{1}{\rho^{1 / 2}} \delta\right\|_{1, h}^{2}=\left\|\frac{1}{\rho^{1 / 2}} \delta\right\|_{0}^{2}+\sum_{j}\left(\frac{1}{\rho} \nabla \delta, \nabla \delta\right)_{j} \\
& =\left\|\frac{1}{\rho^{1 / 2}} \delta\right\|_{0}^{2}+\sum_{j}\left[\left(\frac{1}{\rho} \nabla \delta, \nabla(u-\Pi u)\right)_{j}+\left(\frac{1}{\rho} \nabla \delta, \nabla \eta\right)_{j}\right] \\
& =\left\|\frac{1}{\rho^{1 / 2}} \delta\right\|_{0}^{2}+\sum_{j}\left(\frac{1}{\rho} \nabla \delta, \nabla(u-\Pi u)\right)_{j}+\left(\frac{\omega^{2}}{K} \delta, \eta\right) \\
& +\sum_{j}\left\langle\frac{1}{\rho} \frac{\partial u_{j}}{\partial \nu_{j}}-P_{0} u_{j}, \eta\right\rangle_{\partial \Omega_{j} \backslash \Gamma_{j}}-i \omega\left\langle\left\langle\frac{\alpha}{\rho} \delta, \eta\right\rangle\right\rangle_{\Gamma}-i \omega E\left(\frac{\alpha}{\rho} u, \eta\right) \\
& =\left\|\frac{1}{\rho^{1 / 2}} \delta\right\|_{0}^{2}+\sum_{j}\left(\frac{1}{\rho} \nabla \delta, \nabla(u-\Pi u)\right)_{j}+\left(\frac{\omega^{2}}{K} \delta, \delta\right)-\left(\frac{\omega^{2}}{K} \delta, u-\Pi u\right) \\
& +\sum_{j}\left\langle\frac{1}{\rho} \frac{\partial u_{j}}{\partial \nu_{j}}-P_{0} u_{j}, \eta\right\rangle_{\partial \Omega_{j} \backslash \Gamma_{j}}-i \omega\left\langle\frac{\alpha}{\rho} \delta, \delta\right\rangle_{\Gamma} \\
& +i \omega\left\langle\frac{\alpha}{\rho} \delta, u-\Pi u\right\rangle_{\Gamma}-i \omega E\left(\frac{\alpha}{\rho} u^{h}, \eta\right) .
\end{aligned}
$$


The first four terms in the right side of (3.32) are bounded, for all positive $\varepsilon$, as follows:

$$
\begin{aligned}
& \left|\left\|\frac{1}{\rho^{1 / 2}} \delta\right\|_{0}^{2}+\sum_{j}\left(\frac{1}{\rho} \nabla \delta, \nabla(u-\Pi u)\right)_{j}+\left(\frac{\omega^{2}}{K} \delta, \delta\right)-\left(\frac{\omega^{2}}{K} \delta, u-\Pi u\right)\right| \\
& \quad \leq C(\omega)\left[\|\delta\|_{0}^{2}+\|u-\Pi u\|_{1, h}^{2}\right]+\varepsilon\|\delta\|_{1, h}^{2} \leq C(\omega)\left[\|\delta\|_{0}^{2}+h^{2}\|u\|_{2}^{2}\right]+\varepsilon\|\delta\|_{1, h}^{2} .
\end{aligned}
$$

With properly chosen $q_{j} \in P_{0}\left(\Omega_{j}\right)$ in applying (3.6), the next term in the right side of (3.32) satisfies the estimate

$$
\begin{aligned}
& \left|\sum_{j}\left\langle\frac{1}{\rho} \frac{\partial u_{j}}{\partial \nu_{j}}-P_{0} u_{j}, \eta\right\rangle_{\partial \Omega_{j} \backslash \Gamma_{j}}\right|=\left|\sum_{j}\left\langle\frac{1}{\rho} \frac{\partial u_{j}}{\partial \nu_{j}}-P_{0} u_{j}, \eta_{j}-q_{j}\right\rangle_{\partial \Omega_{j} \backslash \Gamma_{j}}\right| \\
& \quad \leq C h\|u\|_{2}\|\eta\|_{1, h} \leq C h\|u\|_{2}\left[\|\delta\|_{1, h}+\|\Pi u-u\|_{1, h}\right] \leq C\|u\|_{2}^{2} h^{2}+\varepsilon\|\delta\|_{1, h}^{2} .
\end{aligned}
$$

The next two terms in the right side of (3.32) are bounded by using the trace inequality and the approximation properties of $\Pi$ :

$$
\begin{aligned}
& \left|i \omega\left\langle\frac{\alpha}{\rho} \delta, \delta\right\rangle_{\Gamma}-i \omega\left\langle\frac{\alpha}{\rho} \delta, u-\Pi u\right\rangle_{\Gamma}\right| \\
& \quad \leq C(\omega)\left[\|\delta\|_{0}\|\delta\|_{1, h}+\|\delta\|_{1, h}\|u-\Pi u\|_{1, h}\right] \leq C(\omega)\left[\|\delta\|_{0}^{2}+h^{2}\|u\|_{2}^{2}\right]+\varepsilon\|\delta\|_{1, h}^{2} .
\end{aligned}
$$

Finally, by (3.11) the last term in the right side of (3.32) is bounded as follows:

$$
\begin{aligned}
\left|i \omega E\left(\frac{\alpha}{\rho} u^{h}, \eta\right)\right| & \leq C(\omega) h\left\|u^{h}\right\|_{1, h}\|\eta\|_{1, h} \\
& \leq C(\omega) h\left[\|\delta\|_{1, h}+\|u\|_{1}\right]\left[\|\delta\|_{1, h}+\|\Pi u-u\|_{1, h}\right] \\
& \leq C(\omega)\left[h\|\delta\|_{1, h}^{2}+h^{2}\|u\|_{2}^{2}\right]+\varepsilon\|\delta\|_{1, h}^{2} .
\end{aligned}
$$

Substituting (3.33)-(3.36) in (3.32) gives, for sufficiently small $\varepsilon$ and $h$,

$$
\|\delta\|_{1, h} \leq C(\omega)\left[\|\delta\|_{0}+h\|u\|_{2}\right] .
$$

Next, apply (3.37) in (3.31) to obtain

$$
\|\delta\|_{0} \leq C(\omega)\left[h\|\delta\|_{0}+h^{2}\|u\|_{2}+\varepsilon_{\ell}\right],
$$

from which, for sufficiently small $h>0$, it follows that

$$
\|\delta\|_{0} \leq C(\omega)\left[h^{2}\|u\|_{2}+\varepsilon_{\ell}\right] .
$$

For the $H^{1}$-error estimate, insert (3.39) into (3.37) to have

$$
\|\delta\|_{1, h} \leq C(\omega)\left[h\|u\|_{2}+\varepsilon_{\ell}\right] .
$$

We summarize the above results in the following theorem. 
Theorem 3.1 Let $u$ and $u^{h}$ be solutions of (2.1) and (3.8), respectively. Then, for sufficiently small $h>0$ and under the inverse assumption on the partition $\Omega_{j}$,

$$
\begin{aligned}
\left\|u-u^{h}\right\|_{0} & \leq C(\omega)\left[h^{2}\|u\|_{2}+\varepsilon_{\ell}\right], \\
\left\|u-u^{h}\right\|_{1, h} & \leq C(\omega)\left[h\|u\|_{2}+\varepsilon_{\ell}\right],
\end{aligned}
$$

where $\varepsilon_{\ell}$ is defined in (3.25).

Remark 3.1 The inverse assumption is needed only to derive bounds for the error associated with boundary quadrature. If the boundary integrals are evaluated exactly, this assumption can be removed.

Remark 3.2 In the proof of the theorem above, no assumptions on the imaginary parts of $K$ and $\alpha$ were made, though the positivity of $K_{i}$ was used in the existence and uniqueness theorem for the approximate problem. A somewhat more complicated argument would have eliminated the need to require $K_{i}>0$; see [11]. Therefore, Theorem 3.1 holds also for purely real $K$ and $\alpha$.

\section{The Hybridized Procedure}

To hybridize the nonconforming procedure in the manner of Fraeijs de Veubeke [13] and Arnold-Brezzi [3], we employ $\widetilde{\Lambda}^{h}$ as a space of Lagrange multipliers, associating elements $\tilde{\lambda}^{h} \in \widetilde{\Lambda}^{h}$ with $-\frac{1}{\rho} \frac{\partial u^{h}}{\partial \nu_{j k}}\left(\xi_{j k}\right)$ on $\Gamma_{j k}$. We also localize the space $\mathcal{N} \mathcal{C}^{h}$ by introducing the new space

$$
\mathcal{N C}_{-1}^{h}=\left\{v \in L^{2}(\Omega):\left.v\right|_{\Omega_{j}} \in \mathcal{N C}_{j}^{h}\right\}
$$

The hybridized nonconforming procedure then consists in finding $\left(\tilde{u}^{h}, \tilde{\lambda}^{h}\right) \in \mathcal{N C}_{-1}^{h} \times$ $\widetilde{\Lambda}^{h}$ such that

$$
\begin{aligned}
& -\omega^{2}\left(\frac{1}{K(x, \omega)} \tilde{u}^{h}, \varphi\right)+\sum_{j}\left(\frac{1}{\rho} \nabla \tilde{u}^{h}, \nabla \varphi\right)_{j}+i \omega\left\langle\left\langle\frac{\alpha}{\rho} \tilde{u}^{h}, \varphi\right\rangle\right\rangle_{\Gamma} \\
& +\sum_{j, k}\left\langle\left\langle\tilde{\lambda}^{h}, \varphi\right\rangle\right\rangle_{\Gamma_{j k}}=(f, \varphi), \quad \varphi \in \mathcal{N C}_{-1}^{h}, \\
& \sum_{j, k}\left\langle\left\langle\theta, \tilde{u}^{h}\right\rangle\right\rangle_{\Gamma_{j k}}=0, \quad \theta \in \widetilde{\Lambda}^{h} .
\end{aligned}
$$

The following lemma is immediate.

Lemma 4.1 If $\widetilde{u}^{h} \in \mathcal{N C}_{-1}^{h}$, then $\widetilde{u}^{h} \in \mathcal{N} \mathcal{C}^{h}$ if and only if

$$
\sum_{j, k}\left\langle\left\langle\theta, \tilde{u}^{h}\right\rangle\right\rangle_{\Gamma_{j k}}=0, \quad \theta \in \widetilde{\Lambda}^{h} .
$$


The following theorem gives an existence and uniqueness result for the procedure (4.1).

Theorem 4.1 Problem (4.1) has a unique solution which, by Lemma 4.1, coincides with that of (3.8).

Proof. Since (4.1) is finite dimensional, it suffices to show uniqueness. Thus, set $f=0$ in (4.1a), choose $\varphi=\tilde{u}^{h}$ in (4.1a), and use (4.1b) to conclude that

$$
\left((-A+i B) \tilde{u}^{h}, \tilde{u}^{h}\right)+\sum_{j}\left(\frac{1}{\rho} \nabla \tilde{u}^{h}, \nabla \tilde{u}^{h}\right)_{j}+\left\langle\left\langle(C+i D) \tilde{u}^{h}, \tilde{u}^{h}\right\rangle\right\rangle_{\Gamma}=0 .
$$

Taking the imaginary part in (4.2) gives

$$
\left(B \tilde{u}^{h}, \tilde{u}^{h}\right)+\left\langle\left\langle D \tilde{u}^{h}, \tilde{u}^{h}\right\rangle\right\rangle_{\Gamma}=0,
$$

so that $\tilde{u}^{h}=0$. Consequently, (4.1a) reduces to

$$
\sum_{j, k}\left\langle\left\langle\tilde{\lambda}^{h}, \varphi\right\rangle\right\rangle_{\Gamma_{j k}}=0, \quad \varphi \in \mathcal{N} \mathcal{C}_{-1}^{h} .
$$

Next, we show that $\tilde{\lambda}^{h}=0$. Let $\Omega_{j}$ be any element in the partition having a common face $\Gamma_{j k}^{*}$ with another element $\Omega_{k}^{*}$. Then, choose $\varphi=\tilde{\varphi} \in \mathcal{N C}_{-1}^{h}$ such that $\tilde{\varphi}$ is supported in $\Omega_{j}, \tilde{\varphi}\left(\xi_{j k}^{*}\right)=\tilde{\lambda}_{j k}^{*}$ on $\Gamma_{j k}$, and the other degrees of freedom needed to determine $\tilde{\varphi}$ vanish. Then, for any of the suggested quadratures, from (4.3) we conclude that $\tilde{\lambda}_{j k}^{*}=0$; thus, $\tilde{\lambda}_{j k}$ vanishes on all interior boundaries $\Gamma_{j k}$. This completes the proof.

\section{A Domain Decomposition Iterative Procedure}

\subsection{The iterative procedure}

Consider the decomposition of problem (2.1) over the partition $\left\{\Omega_{j}\right\}$. For $j=1, \ldots, J$, find $u_{j}(x, \omega) \in H^{1}\left(\Omega_{j}\right)$ such that

$$
\begin{array}{lll}
\text { i) } & -\frac{\omega^{2}}{K(x, \omega)} u_{j}(x, \omega)-\nabla \cdot\left(\frac{1}{\rho} \nabla u_{j}(x, \omega)\right)=f(x, \omega), & x \in \Omega_{j}, \\
\text { ii) } & \frac{\partial u_{j}(x, \omega)}{\partial \nu_{j}}+i \omega \alpha(x, \omega) u_{j}(x, \omega)=0, & x \in \Gamma_{j},
\end{array}
$$

subject to the interface consistency conditions

$$
\begin{aligned}
u_{j}(x, \omega) & =u_{k}(x, \omega), & & x \in \Gamma_{j k}, \\
\frac{1}{\rho} \frac{\partial u_{j}(x, \omega)}{\partial \nu_{j k}} & =-\frac{1}{\rho} \frac{\partial u_{k}(x, \omega)}{\partial \nu_{k j}}, & & x \in \Gamma_{j k} .
\end{aligned}
$$


Instead of (5.2), we shall impose consistency through the Robin transmission conditions

$$
\begin{array}{ll}
\frac{1}{\rho} \frac{\partial u_{j}}{\partial \nu_{j k}}+\beta_{j k} u_{j}=-\frac{1}{\rho} \frac{\partial u_{k}}{\partial \nu_{k j}}+\beta_{j k} u_{k}, & x \in \Gamma_{j k} \subset \partial \Omega_{j}, \\
\frac{1}{\rho} \frac{\partial u_{k}}{\partial \nu_{k j}}+\beta_{j k} u_{k}=-\frac{1}{\rho} \frac{\partial u_{j}}{\partial \nu_{j k}}+\beta_{j k} u_{j}, & x \in \Gamma_{k j} \subset \partial \Omega_{k},
\end{array}
$$

with $\beta_{j k}$ being a complex-valued function defined on the interfaces $\Gamma_{j k}$.

Since the object of the domain decomposition procedure is to localize the calculations, we motivate our iterative procedure by first defining one at the differential level in the following fashion: Given $u_{j}^{0} \in H^{1}\left(\Omega_{j}\right)$, find $u_{j}^{n} \in H^{1}\left(\Omega_{j}\right)$ such that

$$
\begin{aligned}
& -\omega^{2}\left(\frac{1}{K(x, \omega)} u_{j}^{n}, \varphi\right)_{j}+\left(\frac{1}{\rho} \nabla u_{j}^{n}, \nabla \varphi\right)_{j}+i \omega\left\langle\frac{\alpha}{\rho} u_{j}^{n}, \varphi\right\rangle_{\Gamma_{j}} \\
& \quad+\sum_{k}\left\langle\left[\frac{1}{\rho} \frac{\partial u_{k}^{n-1}}{\partial \nu_{k j}}+\beta_{j k}\left(u_{j}^{n}-u_{k}^{n-1}\right)\right], \varphi\right\rangle_{\Gamma_{j k}}=(f, \varphi)_{j}, \quad \varphi \in H^{1}\left(\Omega_{j}\right) .
\end{aligned}
$$

We shall not pursue an analysis of this iteration but will, instead, define a corresponding domain decomposition iteration for our nonconforming method. Let us introduce a new set $\Lambda^{h}$ of Lagrange multipliers $\lambda_{j k}^{h}$ associated with the fluxes $-\frac{1}{\rho} \frac{\partial u_{j}}{\partial \nu_{j k}}\left(\xi_{j k}\right)$ at the midpoints $\xi_{j k}$ of the interior faces $\Gamma_{j k}$ :

$$
\Lambda^{h}=\left\{\lambda^{h}:\left.\lambda^{h}\right|_{\Gamma_{j k}}=\lambda_{j k}^{h} \in P_{0}\left(\Gamma_{j k}\right) \equiv \Lambda_{j k}\right\}
$$

Note that $\Lambda_{j k}$ and $\Lambda_{k j}$ are considered to be distinct. The iterative procedure is defined as follows. Choose $\left(u_{j}^{h, 0}, \lambda_{j k}^{h, 0}, \lambda_{k j}^{h, 0}\right) \in \mathcal{N C}_{j}^{h} \times \Lambda_{j k} \times \Lambda_{k j}$ arbitrarily. Then, for all $\{j k\}$, compute $\left(u_{j}^{h, n}, \lambda_{j k}^{h, n}\right) \in \mathcal{N C}_{j}^{h} \times \Lambda_{j k}$ as the solution of the equations

$$
\begin{array}{cl}
-\omega^{2}\left(\frac{1}{K(x, \omega)} u_{j}^{h, n}, \varphi\right)_{j}+\left(\frac{1}{\rho} \nabla u_{j}^{h, n}, \nabla \varphi\right)_{j}+i \omega\left\langle\left\langle\frac{\alpha}{\rho} u_{j}^{h, n}, \varphi\right\rangle\right\rangle_{\Gamma_{j}} \\
+\sum_{k}\left\langle\left\langle\lambda_{j k}^{h, n}, \varphi\right\rangle\right\rangle_{\Gamma_{j k}}=(f, \varphi)_{j}, & \varphi \in \mathcal{N C}_{j}^{h}, \\
\lambda_{j k}^{h, n}=-\lambda_{k j}^{h, n-1}+\beta_{j k}\left[u_{j}^{h, n}\left(\xi_{j k}\right)-u_{k}^{h, n-1}\left(\xi_{k j}\right)\right] & \text { on } \Gamma_{j k} .
\end{array}
$$

Equation (5.4a) is useful in the analysis below, but it is implicit in the variable $u^{h, n}$. For computational purposes, (5.4a) should be replaced by

$$
\begin{aligned}
& -\omega^{2}\left(\frac{1}{K(x, \omega)} u_{j}^{h, n}, \varphi\right)_{j}+\left(\frac{1}{\rho} \nabla u_{j}^{h, n}, \nabla \varphi\right)_{j} \\
& +i \omega\left\langle\left\langle\frac{\alpha}{\rho} u_{j}^{h, n}, \varphi\right\rangle\right\rangle_{\Gamma_{j}}+\sum_{k}\left\langle\left\langle\beta_{j k} u_{j k}^{h, n}\left(\xi_{j k}\right), \varphi\right\rangle\right\rangle_{\Gamma_{j k}} \\
& =(f, \varphi)_{j}+\sum_{k}\left\langle\left\langle\lambda_{k j}^{h, n-1}+\beta_{j k} u_{k}^{h, n-1}\left(\xi_{j k}\right), \varphi\right\rangle\right\rangle_{\Gamma_{j k}}, \varphi \in \mathcal{N C}_{j}^{h},
\end{aligned}
$$

after which (5.4b) should be executed. 


\subsection{Convergence of the iterative procedure}

If $\tilde{u}_{j}^{h}=\left.\tilde{u}^{h}\right|_{\Omega_{j}}$ and $\tilde{\lambda}_{j k}^{h}=\left.\tilde{\lambda}^{h}\right|_{\Gamma_{j k}}$, we demonstrate the convergence of $\left(u_{j}^{h, n}, \lambda_{j k}^{h, n}\right)$ to $\left(\tilde{u}_{j}^{h}, \tilde{\lambda}_{j k}^{h}\right)$ as $n$ tends to infinity. For notational simplicity we shall take $\beta_{j k}=\beta_{R}+i \beta_{I}$, $\beta_{R} \geq 0, \beta_{I} \geq 0$; the general case is a trivial extension.

First, note that $\left(\tilde{u}_{j}^{h}, \tilde{\lambda}_{j k}^{h}\right)$ satisfies the local equations

$$
\begin{gathered}
-\omega^{2}\left(\frac{1}{K(x, \omega)} \tilde{u}^{h}, \varphi\right)_{j}+\left(\frac{1}{\rho} \nabla \tilde{u}_{j}^{h}, \nabla \varphi\right)_{j}+i \omega\left\langle\left\langle\frac{\alpha}{\rho} \tilde{u}_{j}^{h}, \varphi\right\rangle\right\rangle_{\Gamma_{j}} \\
+\sum_{k}\left\langle\left\langle\tilde{\lambda}_{j k}^{h}, \varphi\right\rangle\right\rangle_{\Gamma_{j k}}=(f, \varphi)_{j}, \quad \varphi \in \mathcal{N} \mathcal{C}_{j}^{h} .
\end{gathered}
$$

Also, since $\tilde{\lambda}_{j k}^{h}=-\tilde{\lambda}_{k j}^{h},(4.1 \mathrm{~b})$ is equivalent to

$$
\tilde{\lambda}_{j k}^{h}=-\tilde{\lambda}_{k j}^{h}+\beta\left[\tilde{u}_{j}^{h}\left(\xi_{j k}\right)-\tilde{u}_{k}^{h}\left(\xi_{k j}\right)\right] \quad \text { on } \Gamma_{j k} \text {. }
$$

Set

$$
e_{j}^{n}=u_{j}^{h, n}-\tilde{u}_{j}^{h} \text { on } \Omega_{j}, \quad \mu_{j k}^{n}=\lambda_{j k}^{h, n}-\tilde{\lambda}_{j k}^{h} \text { on } \Gamma_{j k} .
$$

From (5.4)-(5.7), we obtain the error equations for the iteration:

$$
\begin{gathered}
-\omega^{2}\left(\frac{1}{K(x, \omega)} e_{j}^{n}, \varphi\right)_{j}+\left(\frac{1}{\rho} \nabla e_{j}^{n}, \nabla \varphi\right)_{j}+i \omega\left\langle\left\langle\frac{\alpha}{\rho} e_{j}^{n}, \varphi\right\rangle\right\rangle_{\Gamma_{j}} \\
+\sum_{k}\left\langle\left\langle\mu_{j k}^{n}, \varphi\right\rangle\right\rangle_{\Gamma_{j k}}=0, \quad \varphi \in \mathcal{N C}_{j}^{h},
\end{gathered}
$$

and

$$
\mu_{j k}^{n}=-\mu_{k j}^{n-1}+\beta\left[e_{j}^{n}\left(\xi_{j k}\right)-e_{k}^{n-1}\left(\xi_{j k}\right)\right], \quad \xi_{j k} \in \Gamma_{j k} .
$$

Choose $\varphi=e_{j}^{n}$ in (5.8) and take the imaginary part in the resulting equation to obtain

$$
\begin{aligned}
& \operatorname{Re} \sum_{k}\left\langle\left\langle\mu_{j k}^{n}, e_{j}^{n}\right\rangle\right\rangle_{\Gamma_{j k}}=\left(A e_{j}^{n}, e_{j}^{n}\right)_{j}-\left(\frac{1}{\rho} \nabla e_{j}^{n}, \nabla \varphi\right)_{j}-\left\langle\left\langle C e_{j}^{n}, e_{j}^{n}\right\rangle\right\rangle_{\Gamma_{j}}, \\
& \operatorname{Im} \sum_{k}\left\langle\left\langle\mu_{j k}^{n}, e_{j}^{n}\right\rangle\right\rangle_{\Gamma_{j k}}=-\left(B e_{j}^{n}, e_{j}^{n}\right)_{j}-\left\langle\left\langle D e_{j}^{n}, e_{j}^{n}\right\rangle\right\rangle_{\Gamma_{j}} .
\end{aligned}
$$

Since, for any pair of complex numbers $p$ and $q$,

$$
\begin{gathered}
|p \pm \beta q|^{2}=|p|^{2}+|\beta|^{2}|q|^{2} \pm 2\left[\beta_{R} \operatorname{Re}(p \bar{q})+\beta_{I} \operatorname{Im}(p \bar{q})\right], \\
\sum_{j} \sum_{k}\left|\mu_{j k}^{n} \pm \beta e_{j}^{n}\left(\xi_{j k}\right)\right|_{0, \Gamma_{j k}}^{2}=\sum_{j, k}\left[\left|\mu_{j k}^{n}\right|_{0, \Gamma_{j k}}^{2}+|\beta|^{2}\left|e_{j}^{n}\left(\xi_{j k}\right)\right|_{0, \Gamma_{j k}}^{2}\right] \\
\mp 2 \beta_{R} \sum_{j}\left[-\left(A e_{j}^{n}, e_{j}^{n}\right)_{j}+\left(\frac{1}{\rho} \nabla e_{j}^{n}, \nabla e_{j}^{n}\right)_{j}+\left\langle\left\langle C e_{j}^{n}, e_{j}^{n}\right\rangle\right\rangle_{\Gamma_{j}}\right] \\
\mp 2 \beta_{I} \sum_{j}\left[\left(B e_{j}^{n}, e_{j}^{n}\right)_{j}+\left\langle\left\langle D e_{j}^{n}, e_{j}^{n}\right\rangle\right\rangle_{\Gamma_{j}}\right] .
\end{gathered}
$$


Set

$$
R^{n}=R\left(e^{n}, \mu^{n}\right)=\sum_{j} \sum_{k}\left|\mu_{j k}^{n}-\beta e_{j}^{n}\left(\xi_{j k}\right)\right|_{0, \Gamma_{j k}}^{2} .
$$

Then, (5.9) and (5.10) imply that

$$
\begin{aligned}
& R^{n}= \sum_{k} \sum_{j}\left|\mu_{k j}^{n-1}+\beta e_{k}^{n-1}\left(\xi_{j k}\right)\right|_{0, \Gamma_{k j}}^{2} \\
&= \sum_{k, j}\left[\left|\mu_{k j}^{n-1}\right|_{0, \Gamma_{k j}}^{2}+|\beta|^{2}\left|e_{k}^{n-1}\left(\xi_{j k}\right)\right|_{0, \Gamma_{k j}}^{2}\right] \\
& \quad-2 \beta_{R} \sum_{k}\left[-\left(A e_{k}^{n-1}, e_{k}^{n-1}\right)_{k}+\left(\frac{1}{\rho} \nabla e_{k}^{n-1}, \nabla e_{k}^{n-1}\right)_{k}+\left\langle\left\langle C e_{k}^{n-1}, e_{k}^{n-1}\right\rangle\right\rangle_{\Gamma_{k}}\right] \\
& \quad-2 \beta_{I} \sum_{k}\left[\left(B e_{k}^{n-1}, e_{k}^{n-1}\right)_{k}+\left\langle\left\langle D e_{k}^{n-1}, e_{k}^{n-1}\right\rangle\right\rangle_{\Gamma_{k}}\right] \\
&= R^{n-1}-4 \beta_{R} \sum_{j}\left[-\left(A e_{j}^{n-1}, e_{j}^{n-1}\right)_{j}+\left(\frac{1}{\rho} \nabla e_{j}^{n-1}, \nabla e_{j}^{n-1}\right)_{j}+\left\langle\left\langle C e_{j}^{n-1}, e_{j}^{n-1}\right\rangle\right\rangle_{\Gamma_{j}}\right] \\
& \quad-4 \beta_{I} \sum_{j}\left[\left(B e_{j}^{n-1}, e_{j}^{n-1}\right)_{j}+\left\langle\left\langle D e_{j}^{n-1}, e_{j}^{n-1}\right\rangle\right\rangle_{\Gamma_{j}}\right] \leq R^{n-1},
\end{aligned}
$$

provided that $\beta_{I} B_{\min }-\beta_{R} A_{\max }>0$. Therefore, under this condition on the coefficient $\beta$,

$$
\begin{aligned}
R^{n}=R^{0}- & 4 \beta_{R} \sum_{m=0}^{n-1} \sum_{j}\left[-\left(A e_{j}^{m-1}, e_{j}^{m-1}\right)_{j}+\left(\frac{1}{\rho} \nabla e_{j}^{m-1}, \nabla e_{j}^{m-1}\right)_{j}+\left\langle\left\langle C e_{j}^{m-1}, e_{j}^{m-1}\right\rangle\right\rangle_{\Gamma_{j}}\right] \\
& -4 \beta_{I} \sum_{m=0}^{n-1} \sum_{j}\left[\left(B e_{j}^{m-1}, e_{j}^{m-1}\right)_{j}+\left\langle\left\langle D e_{j}^{m-1}, e_{j}^{m-1}\right\rangle\right\rangle_{\Gamma_{j}}\right] \geq 0,
\end{aligned}
$$

and the sequence $\left\{R^{n}\right\}$ converges. From the assumed relation $\beta_{I} B_{\min }-\beta_{R} A_{\max }>0$ and the positivity of $C$ and $D$, we can conclude that $e_{j}^{n}$ tends to zero when $n \rightarrow \infty$. Note that the convergence of $\left\{R^{n}\right\}$ also implies that $\left|\mu_{j k}^{n}\right|$ is bounded independently of $n, j$, and $k$. Then, choosing $\varphi$ in (5.8) equal to $\mu_{j k}^{n}$ at $\xi_{j k}$ and zero at the other nodes on $\Omega_{j}$ shows that $\mu_{j k}^{n} \rightarrow 0$. Therefore, $R^{n} \rightarrow 0$ as $n \rightarrow \infty$.

\subsection{An estimate for the spectral radius of the iterative proce- dure}

We shall give a second, and more precise, demonstration of the convergence of the iterative procedure (5.4) by showing that the iterations approach the fixed point of an operator $T_{f}$ defined as follows. Given $f \in L^{2}(\Omega)$, let $T_{f}: N C_{-1}^{h} \times \Lambda^{h} \rightarrow N C_{-1}^{h} \times \Lambda^{h}$ be 
the affine map such that for any $(p, \theta) \in N C_{-1}^{h} \times \Lambda^{h},(u, \lambda)=T_{f}(p, \theta)$ is the solution of the equations

$$
\begin{aligned}
& -\omega^{2}\left(\frac{1}{K(x, \omega)} u_{j}, \varphi\right)_{j}+\left(\frac{1}{\rho} \nabla u_{j}, \nabla \varphi\right)_{j}+i \omega\left\langle\left\langle\frac{\alpha}{\rho} u_{j}, \varphi\right\rangle\right\rangle_{\Gamma_{j}} \\
& \quad+\sum_{k}\left\langle\left\langle\beta u_{j}\left(\xi_{j k}\right), \varphi\right\rangle_{\Gamma_{j k}}=(f, \varphi)_{j}+\sum_{k}\left\langle\left\langle\theta_{k j}+\beta p_{k}\left(\xi_{j k}\right), \varphi\right\rangle\right\rangle_{\Gamma_{j k}}, \quad \varphi \in \mathcal{N C}_{j}^{h},\right. \\
& \lambda_{j k}=-\theta_{k j}+\beta\left[u_{j}\left(\xi_{j k}\right)-p_{k}\left(\xi_{j k}\right)\right], \quad \xi_{j k} \in \Gamma_{j k} .
\end{aligned}
$$

Lemma 5.1 The pair $(u, \lambda)$ is a solution of (5.6)-(5.7) if and only if it is a fixed point of $T_{f}$. If $(u, \lambda)$ is a fixed point of $T_{f}$, then $u_{j}\left(\xi_{j k}\right)=u_{k}\left(\xi_{j k}\right)$ and $\lambda_{j k}=-\lambda_{j k}$ for all $j, k$.

Proof. Let $(u, \lambda)$ be a fixed point of $T_{f}$. By (5.13),

$$
-\omega^{2}\left(\frac{1}{K(x, \omega)} u_{j}, \varphi\right)_{j}+\left(\frac{1}{\rho} \nabla u_{j}, \nabla \varphi\right)_{j}+i \omega\left\langle\left\langle\frac{\alpha}{\rho} u_{j}, \varphi\right\rangle\right\rangle_{\Gamma_{j}}+\sum_{k}\left\langle\left\langle\lambda_{j k}, \varphi\right\rangle\right\rangle_{\Gamma_{j k}}=(f, \varphi)_{j},
$$

so that $(u, \lambda)$ satisfies $(5.6)$. Next, from $(5.13 b)$,

$$
\lambda_{j k}=-\lambda_{k j}+\beta\left(u_{j}\left(\xi_{j k}\right)-u_{k}\left(\xi_{j k}\right)\right)
$$

and (5.7) is satisfied. Also, from (5.13b),

$$
\lambda_{k j}=-\lambda_{j k}+\beta\left(u_{k}\left(\xi_{j k}\right)-u_{j}\left(\xi_{j k}\right)\right) .
$$

From the last two equations we conclude that $u_{j}\left(\xi_{j k}\right)=u_{k}\left(\xi_{j k}\right)$ and from (5.14) we have $\lambda_{j k}=-\lambda_{j k}$, as desired. This proves one of the conclusions. The other implication follows immediately from the fact that any solution of (5.6)-(5.7) is a fixed point of $T_{f}$. This completes the proof.

Next, let $\left(u^{0}, \lambda^{0}\right)=T_{0}(p, \theta)$ be the solution of (5.6)-(5.7) for $f=0$, so that $T_{f}(p, \theta)=T_{0}(p, \theta)+T_{f}(0,0)$ and $(p, \theta)$ is a fixed point of $T_{f}$ if and only if

$$
T_{f}(p, \theta)=(p, \theta)=T_{0}(p, \theta)+T_{f}(0,0),
$$

so that a fixed point of $T_{f}$ is a solution of the equation

$$
\left(I-T_{0}\right)(p, \theta)=T_{f}(0,0) .
$$

In the analysis of these fixed points and in the remainder of the paper, the following notation is convenient:

$$
h_{\max }=\max _{j}\left\{h_{\max }\left(\Omega_{j}\right)\right\}, \quad h_{\min }=\min _{j}\left\{h_{\min }\left(\Omega_{j}\right)\right\}, \quad \zeta=\max _{j} \frac{h_{\max }\left(\Omega_{j}\right)}{h_{\min }\left(\Omega_{j}\right)},
$$

where $h_{\min }\left(\Omega_{j}\right)$ and $h_{\max }\left(\Omega_{j}\right)$ denote the maximum diameter of $\Omega_{j}$ and minimum diameter of a ball inscribed in $\Omega_{j}$. 
Theorem 5.1 Assume that $\beta$ is chosen such that $\beta_{I} B_{\min }-\beta_{R} A_{\max }>0, \beta_{R} \geq 0, \beta_{I} \geq$ 0 . Let $\rho\left(T_{0}\right)$ be the spectral radius of $T_{0}$. Then there exists a positive constant $M=$ $M(\beta)$ such that

$$
\rho^{2}\left(T_{0}\right)=1-\frac{1}{M} \equiv \gamma_{0}^{2} ;
$$

consequently, the iterative procedure (5.4) is convergent with an error in the $n^{\text {th }}$-iteration bounded by $O\left(\gamma_{0}^{n}\right)$. Moreover,

$$
\gamma_{0}^{2} \leq 1-C h_{\min },
$$

with $C>0$ being a computable constant depending only upon the medium and $\beta$.

Proof. Let $\gamma$ be an eigenvalue of $T_{0}$ with associated eigenvector $(u, \lambda)$, so that $T_{0}(u, \lambda)=\gamma(u, \lambda)$. By $(5.11)$,

$$
R\left(T_{0}(u, \lambda)\right)=|\gamma|^{2} R(u, \lambda),
$$

and, by (5.12),

$$
\begin{aligned}
R\left(T_{0}(u, \lambda)\right)=R(u, \lambda) & -4 \beta_{R} \sum_{j}\left[-\left(A u_{j}, u_{j}\right)_{j}+\left(\frac{1}{\rho} \nabla u_{j}, \nabla u_{j}\right)_{j}+\left\langle\left\langle C u_{j}, u_{j}\right\rangle\right\rangle_{\Gamma_{j}}\right] \\
& -4 \beta_{I} \sum_{j}\left[\left(B u_{j}, u_{j}\right)_{j}+\left\langle\left\langle D u_{j}, u_{j}\right\rangle\right\rangle_{\Gamma_{j}}\right] .
\end{aligned}
$$

Combining (5.17) and (5.18) gives

$$
\begin{aligned}
|\gamma|^{2}=1 & -4 \beta_{R} \sum_{j}\left[-\left(A u_{j}, u_{j}\right)_{j}+\left(\frac{1}{\rho} \nabla u_{j}, \nabla u_{j}\right)_{j}+\left\langle\left\langle C u_{j}, u_{j}\right\rangle\right\rangle_{\Gamma_{j}}\right] / R(u, \lambda) \\
& -4 \beta_{I} \sum_{j}\left[\left(B u_{j}, u_{j}\right)_{j}+\left\langle\left\langle D u_{j}, u_{j}\right\rangle\right\rangle_{\Gamma_{j}}\right] / R(u, \lambda) \\
\leq 1 & -4 \min \left(\beta_{I} B_{\min }-\beta_{R} A_{\max } \frac{\beta_{R}}{\rho_{\max }}\right)\left[\|u\|_{0}^{2}+\|\nabla u\|_{0, h}^{2}\right] / R(u, \lambda),
\end{aligned}
$$

so that $|\gamma| \leq 1$.

Now, $R(u, \lambda)$ will be estimated in terms of the sizes and shapes of the subdomains to obtain a rate of convergence of the iteration (5.4), which can be interpreted as a domain decomposition procedure at the level of individual elements.

It follows from (5.13) that

$$
\begin{gathered}
-\omega^{2}\left(\frac{1}{K(x, \omega)} u_{j}, \varphi\right)_{j}+\left(\frac{1}{\rho} \nabla u_{j}, \nabla \varphi\right)_{j}+i \omega\left\langle\left\langle\frac{\alpha}{\rho} u_{j}, \varphi\right\rangle\right\rangle_{\Gamma_{j}}+\sum_{k}\left\langle\left\langle\lambda_{j k}, \varphi\right\rangle\right\rangle_{\Gamma_{j k}} \\
=0, \quad \varphi \in \mathcal{N C}_{j}^{h} .
\end{gathered}
$$


Let $\Omega_{j}$ be any element and let $\Gamma_{j k}^{*}$ be an interior face of $\Omega_{j}$ common with another element $\Omega_{k}$. Choose $\varphi=\tilde{\varphi} \in N C_{j}^{h}$ in (5.20) such that $\tilde{\varphi}\left(\xi_{j k}^{*}\right)=\lambda_{j k}^{*}$ on $\Gamma_{j k}^{*}$ and all other degrees of freedom needed to determine $\tilde{\varphi}$ vanish. Scaling arguments show that

$$
\begin{aligned}
& \|\tilde{\varphi}\|_{0, \Omega_{j}}^{2} \leq C h_{\max }\left(\Omega_{j}\right)\left\langle\left\langle\lambda_{j k}^{*}, \lambda_{j k}^{*}\right\rangle\right\rangle_{\Gamma_{j k}^{*}}, \\
& \|\nabla \tilde{\varphi}\|_{0, \Omega_{j}}^{2} \leq C \frac{h_{\max }\left(\Omega_{j}\right)}{h_{\min }\left(\Omega_{j}\right)^{2}}\left\langle\left\langle\lambda_{j k}^{*}, \lambda_{j k}^{*}\right\rangle\right\rangle_{\Gamma_{j k}^{*}} .
\end{aligned}
$$

Thus,

$$
\begin{aligned}
& \left\langle\left\langle\lambda_{j k}^{*}, \lambda_{j k}^{*}\right\rangle\right\rangle_{\Gamma_{j k}^{*}}=\left(\frac{\omega^{2}}{K(x, \omega)} u_{j}, \tilde{\varphi}\right)_{j}-\left(\frac{1}{\rho} \nabla u_{j}, \nabla \tilde{\varphi}\right)_{j} \\
& \leq C h_{\max }\left(\Omega_{j}\right)^{1 / 2}\left[\left\|u_{j}\right\|_{0, \Omega_{j}}+h_{\min }\left(\Omega_{j}\right)^{-1}\left\|\nabla u_{j}\right\|_{0, \Omega_{j}}\right]\left[\left\langle\left\langle\lambda_{j k}^{*}, \lambda_{j k}^{*}\right\rangle\right\rangle_{\Gamma_{j k}^{*}}\right]^{1 / 2}
\end{aligned}
$$

from which we can conclude that, for all elements $\Omega_{j}$,

$$
\sum_{k}\left\langle\left\langle\lambda_{j k}, \lambda_{j k}\right\rangle\right\rangle_{\Gamma_{j k}} \leq C h_{\max }\left(\Omega_{j}\right)\left[\left\|u_{j}\right\|_{0, \Omega_{j}}^{2}+h_{\min }\left(\Omega_{j}\right)^{-2}\left\|\nabla u_{j}\right\|_{0, \Omega_{j}}^{2}\right] .
$$

It was shown for rectangular case in [12] that

$$
\left\langle\left\langle u_{j}, u_{j}\right\rangle\right\rangle_{\Gamma_{j k}} \leq C h_{\min }\left(\Omega_{j}\right)^{-1}\left\|u_{j}\right\|_{0, \Omega_{j}}^{2} ;
$$

for the triangular case, the estimate follows from the triangle inequality and the inverse inequality combined with (3.11). Combining (5.23), (5.24), and the inverse inequality $\left\|\nabla u_{j}\right\|_{0, \Omega_{j}} \leq C h_{\min }\left(\Omega_{j}\right)^{-1}\left\|u_{j}\right\|_{0, \Omega_{j}}$ gives

$$
\begin{aligned}
R(u, \lambda)= & \sum_{k} \sum_{j}\left|\lambda_{j k}-\beta u_{j}\left(\xi_{j k}\right)\right|_{0, \Gamma_{k j}}^{2} \leq 2 \sum_{j, k}\left[\left|\lambda_{j k}\right|_{0, \Gamma_{k j}}^{2}+|\beta|^{2}\left\langle\left\langle u_{j}, u_{j}\right\rangle\right\rangle_{\Gamma_{j k}}\right] \\
\leq & C \sum_{j} h_{\min }^{-1}\left(\Omega_{j}\right)\left[h_{\max }\left(\Omega_{j}\right) h_{\min }\left(\Omega_{j}\right)\left(\left\|u_{j}\right\|_{0, \Omega_{j}}^{2}+h_{\min }\left(\Omega_{j}\right)^{-2}\left\|\nabla u_{j}\right\|_{0, \Omega_{j}}^{2}\right)\right. \\
& \left.\quad \quad \quad|\beta|^{2}\left\|u_{j}\right\|_{0, \Omega_{j}}^{2}\right] \\
\leq & C h_{\min }^{-1} \sum_{j}\left[\left(h_{\max }\left(\Omega_{j}\right) h_{\min }\left(\Omega_{j}\right)+|\beta|^{2}\right)\left\|u_{j}\right\|_{0, \Omega_{j}}^{2}+\zeta\left\|\nabla u_{j}\right\|_{0, \Omega_{j}}^{2}\right] \\
\leq & C h_{\min }^{-1} \max \left(h_{\max }^{2}+|\beta|^{2}, \zeta\right)\left[\|u\|_{0}^{2}+\|\nabla u\|_{0, h}^{2}\right],
\end{aligned}
$$

with $h_{\max }, h_{\min }$, and $\zeta$ as defined in (5.16).

Now combining (5.19) and (5.25) we conclude that

$$
\rho^{2}\left(T_{0}\right) \leq 1-\frac{4 \min \left(\beta_{I} B_{\min }-\beta_{R} A_{\max }, \beta_{R} / \rho_{\max }\right)}{C \max \left(h_{\max }^{2}+|\beta|^{2}, \zeta\right)} h_{\min }=1-N(\beta) h_{\min },
$$

as desired. This completes the proof. 


\section{Appendix}

Here, we prove an estimate for the boundary quadrature error employed in the derivation of the error estimates for the procedure (3.8).

Lemma 6.1 Assume the partition $\left\{\Omega_{j}\right\}$ to be quasiregular. Let $\mathcal{N C}_{\ell}^{h}$ denote the nonconforming space corresponding to the use of $\theta_{\ell}$ in the definition of its local basis. Then, for $\varphi, \psi \in \mathcal{N C}_{\ell}^{h}$,

$$
\left|E_{\ell}(\varphi, \psi)\right| \leq C h\|\varphi\|_{1, h}\|\psi\|_{1, h}, \quad \ell=1 \text { or } 2 .
$$

Proof. Let $F$ be a face (or a side) of $\Omega_{j}$ of diameter $h$, and let $\varphi, \psi \in \mathcal{N C}_{j}^{h}$. Consider first the case $\ell=1$. Then,

$$
\begin{aligned}
\left|\langle\varphi, \psi\rangle_{F}-\langle\langle\varphi, \psi\rangle\rangle_{F}\right| & =\left|\langle\varphi, \psi\rangle_{F}-\varphi(\xi) \overline{\psi(\xi)}\right| F||=\left|\langle\varphi-\varphi(\xi), \psi-\psi(\xi)\rangle_{F}\right| \\
& \leq C h^{2}|\varphi|_{1, F}|\psi|_{1, F} \\
& \leq C h^{2}\|\varphi\|_{1, \Omega_{j}}^{1 / 2}\|\varphi\|_{2, \Omega_{j}}^{1 / 2}\|\psi\|_{1, \Omega_{j}}^{1 / 2}\|\psi\|_{2, \Omega_{j}}^{1 / 2} \\
& \leq C h\|\varphi\|_{1, \Omega_{j}}\|\psi\|_{1, \Omega_{j}},
\end{aligned}
$$

where the orthogonality property (3.6), the quadrature errors on $F$, a trace inequality, and an inverse inequality were invoked. Therefore, in general, for $\varphi, \psi \in \mathcal{N C}_{j}^{h}$,

$$
\left|\langle\varphi, \psi\rangle_{F}-\langle\langle\varphi, \psi\rangle\rangle_{F}\right| \leq C h\|\varphi\|_{1, h}\|\psi\|_{1, h},
$$

so that the lemma follows for $\ell=1$.

For $\ell=2$, let $F$ denote the face $z=1$ of the reference cube and decompose $\varphi$ and $\psi$ as follows;

$$
\varphi=\varphi_{1}+\varphi_{2}, \quad \varphi_{1} \in \operatorname{Span}\{1, x, y\}, \quad \varphi_{2} \in \operatorname{Span}\left\{\theta_{2}(x), \theta_{2}(y)\right\},
$$

with $\psi_{1}$ and $\psi_{2}$ defined analogously. Then,

$$
\langle\varphi, \psi\rangle_{F}=\left\langle\varphi_{1}, \psi_{1}\right\rangle_{F}+\left\langle\varphi_{2}, \psi_{2}\right\rangle_{F}
$$

since the two subspaces are orthogonal. By the construction of $\theta_{2}$,

$$
\langle\langle\varphi, \psi\rangle\rangle_{F}=\left\langle\left\langle\varphi_{1}, \psi_{1}\right\rangle\right\rangle_{F}
$$

so that

$$
\langle\varphi, \psi\rangle_{F}-\langle\langle\varphi, \psi\rangle\rangle_{F}=\left\langle\varphi_{2}, \psi_{2}\right\rangle_{F}
$$

Now, the best linear interpolants of $\varphi_{2}$ and $\psi_{2}$ are identically zero; hence,

$$
\left|\langle\varphi, \psi\rangle_{F}-\langle\langle\varphi, \psi\rangle\rangle_{F}\right| \leq C\left|\varphi_{2}\right|_{0, F}\left|\psi_{2}\right|_{0, F} \leq C|\varphi|_{0, F}|\psi|_{0, F},
$$

again applying the orthogonality of the two subspaces. Scaling and a trace inequality lead to the conclusion for $\ell=2$. 


\section{References}

[1] A. Adini and R. W. Clough. Analysis of plate bending by the finite element method. NSF Report G. 7337 (1961).

[2] D. N. Arnold, D. Boffi, and R. S. Falk. Approximation by quadrilateral finite elements. To appear; currently available by anonymous ftp.math.psu.edu as pub/dna/papers/quadapprox.ps.

[3] D. N. Arnold and F. Brezzi. Mixed and nonconforming finite element methods: implementation, postprocessing and error estimates. R.A.I.R.O. Modélisation Mathématique et Analyse Numérique, 19 (1985), pp. 7-32.

[4] S. C. Brenner and L. R. Scott. "The Mathematical Theory of Finite Element Methods", Springer-Verlag, New York, 1994.

[5] Z. Cai, J. Douglas, Jr., J. E. Santos, D. Sheen, and X. Ye. Nonconforming quadrilateral finite elements: A correction. To appear in Calcolo.

[6] P. G. Ciarlet. "The Finite Element Method for Elliptic Equations", North-Holland, Amsterdam, 1978.

[7] M. Crouzeix and P.-A. Raviart. Conforming and nonconforming finite element methods for solving the stationary Stokes equations. R.A.I.R.O. Modélisation Mathématique et Analyse Numérique, 3 (1973), pp. 33-75.

[8] B. Després. "Méthodes de décomposition de domaines pour les problèmes de propagation d'ondes en régime harmonique", Thèse, Université Paris IX Dauphine, UER Mathématiques de la Décision, 1991.

[9] J. Douglas, Jr., P. J. Paes Leme, J. E. Roberts, and J. Wang. A parallel iterative procedure applicable to the approximate solution of second order partial differential equations by mixed finite element methods. Numer. Math., 65 (1993), pp. 95-108.

[10] J. Douglas, Jr., J. E. Santos, D. Sheen and L. S. Bennethum. Frequency domain treatment of one-dimensional scalar waves. Mathematical Models and Methods in the Applied Sciences, 3 (1993), pp. 171-194.

[11] J. Douglas, Jr., J. E. Santos and D. Sheen. Approximation of scalar waves in the space-frequency domain. Mathematical Models and Methods in the Applied Sciences, 4 (1994), pp. 509-531.

[12] J. Douglas, Jr., J. E. Santos, D. Sheen and X. Ye. Nonconforming Galerkin Methods bases on Quadrilateral Elements for Second Order Elliptic Problems. Mathematical Modelling and Numerical Analysis, 33 (1999), pp. 747-770.

[13] B. X. Fraeijs de Veubeke. Stress function approach. International Congress on the Finite Element Method in Structual Mechanics, Bournemouth, 1975. 
[14] P. M. Gauzellino, J. E. Santos, and D. Sheen. Frequency domain wave propagation modelling in exploration seismology. To appear in the Proceedings of ICTA'99: Fourth International Conference on Theoretical and Computational Acoustics, Stazione Maritima, Trieste, Italy, May 10-14, 1999.

[15] J. Kim and D. Sheen. An Elliptic Regularity of a Helmholtz-Type Problem with an Absorbing Boundary Condition. Bull. Korean Math. Soc. 34 (1997), pp. 135-146.

[16] P. L. Lions. On the Schwarz alternating method, I. In "Domain Decomposition Methods for Partial Differential Equations", R. Glowinski, G. Golub, G. Meurant, and J. Periaux, eds., pages 1-42, Philadelphia, SIAM, 1988.

[17] P. L. Lions. On the Schwarz alternating method III: a variant for nonoverlapping subdomains. In "Domain Decomposition Methods for Partial Differential Equations", T. F. Chan, R. Glowinski, J. Periaux, and O. B. Widlund, eds., pages 202-223, SIAM, Philadelphia, PA, 1990.

[18] H. P. Liu, D. L. Anderson, and H. Kanamori Velocity dispersion due to anelasticity; implications for seismology and mantle composition. Geophys. J. Roy. Astr. Soc., 47 (1976), pp. 41-58.

[19] L. S. D. Morley The triangular equilibrium element in the solution of plate bending problems. Aero. Quart., 19 (1968), pp. 149-169.

[20] R. Rannacher and S. Turek Simple nonconforming quadrilateral Stokes element. Numerical Methods for Partial Differential Equations, 8 (1992), pp. 97-111.

[21] C. L. Ravazzoli and J. E. Santos. Consistency analysis for a model for wave propagation in anelastic media. Latin American Applied Research, 25 (1995), pp. 141-152.

[22] A. H. Schatz. An observation concerning Ritz-Galerkin methods with indefinite bilinear forms. Math. Comp., 28 (1974), pp. 959-962.

[23] G. Strang. Variational crimes in the finite element method. In "The Mathematical Foundations of the Finite Element Method with Applications to Partial Differential Equations", pages 689-710. Academic Press, New York, 1972. A. K. Aziz, ed.

[24] G. Strang and G. J. Fix. "An Analysis of the Finite Element Method", PrenticeHall, Englewood Cliffs, 1973.

[25] O. C. Zienkiewicz, R. L. Taylor, and J. M. Too Reduced integration technique in general analysis of plates and shells. Int. J. Numer. Methods Engr., 3 (1971), pp. 275-290. 